\title{
Heavy Quark Production at CDF
}

\author{
Ch. Paus ${ }^{\mathrm{a}}$ for the CDF Collaboration \\ a Massachusetts Institute of Technology, 77 Massachusetts Avenue, Cambridge, MA 02139, USA
}

The contribution summarizes the latest results from CDF on heavy quark production. Results from top, bottom and charm production are included. Some new analysis using Run I (1991-1994) data have become available. More importantly there are a number of results using Run II data which began in April 2001. The data indicate the potential of CDF for bottom and charm production physics in the near future.

\section{INTRODUCTION}

The experiments at the Tevatron are, until the LHC turns on, in the unique position to study all quarks which are considered heavy: charm, bottom, and top. In the following a number of recent results from CDF are presented which are obtained from data of the Run I and Run II data taking periods. For heavy quark physics at hadron machines like the Tevatron the triggering system is crucial. Most of the collisions are uninteresting with relatively low momenta going in the transverse direction and originate from light quarks. Interesting events have to be filtered out carefully due to limited bandwidth.

During the Run I data taking period (1991-4) CDF took a total of $110 \mathrm{pb}^{-1}$ of data. These data are used extensively for analysis and even today new results of heavy quark production are being obtained. For $b$ physics analyses data originating from muon and electron triggers are used.

The Run II data taking period started in April 2001 and CDF has used up to $15 \mathrm{pb}^{-1}$ for the analyses presented here. The most exciting new feature of the CDF detector with respect to the Run I setup is the trigger for large impact parameter tracks [1]. For the first time at a hadron machine a level-2 trigger is able to measure the track impact parameter fast and accurately enough to allow a trigger based on these measurements. This opens a new window of opportunity for heavy flavor physics based on purely hadronic decays. This trigger will be essential to measure $B_{s}$ mixing, which is one of the most important measurements targeted by CDF in the near term fu- ture. Probably only the fully reconstructed final state leads to sufficiently precise proper time measurements to capture the rapid $B_{s}$ oscillations.

Heavy quark production is predicted by QCD and the larger the mass, the better the agreement with perturbative calculations. One of the main goals in measuring heavy quark production is to test the validity of QCD calculation schemes. The three quarks cover an interesting spectrum from the top quark mass of about $175 \mathrm{GeV} / c^{2}$ to the charm quark mass of about $1.5 \mathrm{GeV} / c^{2}$.

The text summarizes recent results for top and bottom production in the next two sections. Both are based on Run I data. The next section, number 4 , is devoted to Run II data, first showing the detector performance in the leptonic triggers and then some first results on charm decays using the new hadronic trigger.

\section{RECENT RESULTS FROM TOP PRODUCTION}

Gluon radiation is an important aspect of top quark production. Not only is the phenomenon interesting in itself but it also constitutes a large component of the uncertainty of the top quark mass measurement. While the total uncertainty on the top quark mass is about $\pm 5 \mathrm{GeV} / c^{2}$ in Run I, the uncertainty introduced by hard gluon radiation amounts to $\pm 2-3 \mathrm{GeV} / c^{2}$ depending on the particular top decay channel.

Hard gluon radiation can in good approximation be separated into gluon radiation in production which means before the top quark decays, and gluon radiation in decay which happens dur- 
ing or after the top decay. While jets reconstructed from the former type should not be included in the $t \bar{t}$ system the latter one has to be included to obtain the correct top quark mass.

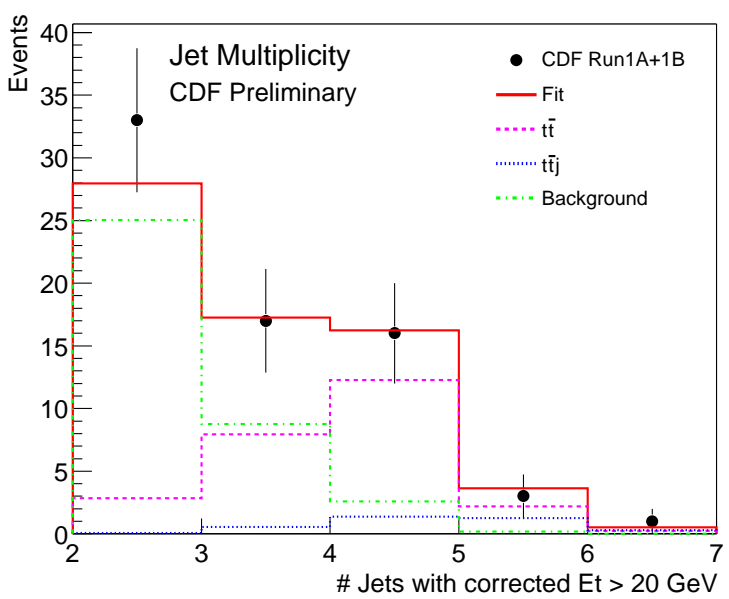

Figure 1. Fit of the Monte Carlo jet multiplicity templates to the data.

The $t \bar{t}$ is reconstructed in the Run I data as a lepton plus missing energy and at least two jets [2]. At least one of the jets is required to have a $b$-tag which means that it contains tracks originating from a secondary vertex. For the analysis the events are classified as $t \bar{t}$ plus $0,1,2$ etc. jets. Jets are formed based on a cone algorithm [2] using different transverse energy thresholds: $E_{T}>20 \mathrm{GeV}$ or $E_{T}>25 \mathrm{GeV}$. The jet multiplicity is then fit to Monte Carlo templates. The Monte Carlo uses Pythia [3], where gluon radiation in production is included via MADGRAPH matrix elements [4]. The processes $q q / g g \rightarrow t t$, $q q / g g \rightarrow t t g, q g \rightarrow t t q$, and $q g \rightarrow t t g g$ are included. Gluon radiation in the top decay is provided by Pythia itself. Using templates of the jet multiplicities of the signal Monte Carlo and the standard background simulation [2] allows one to determine the contribution of the different gluon radiation diagrams. A fit of the jet multiplicity templates for jets with transverse energy larger $E_{T}>20 \mathrm{GeV}$ to the data is shown in Figure 1.
Table 1

Fraction of events originating from background, $t \bar{t}$ or $t \bar{t} j$ production.

\begin{tabular}{lc}
\hline Contribution & Fit $\left(E_{T}>20 \mathrm{GeV}\right)$ \\
\hline$f_{t t}$ & $0.36_{-0.12}^{+0.13}$ \\
$f_{t t j}$ & $0.051_{-0.059}^{+0.084}$ \\
$f_{b g}$ & $0.523_{-0.056}^{+0.057}$ \\
\hline
\end{tabular}

For events with only 2 jets the background dominates while the fraction of top-pair events without radiation increases until jet multiplicities of 4. The contribution of top-pairs with one hard gluon radiation reaches its maximum at 4 to 5 jets. The result of the fit is the fraction of $t \bar{t}$, and $t \bar{t} j$ events: $f_{t t}$ and $f_{t t j}$, respectively, and is listed in Table 1.

The large uncertainty on the fraction of $t \bar{t} j$ events indicates that the number of such events is not yet sufficient to make a significant measurement of this process. A fit including an additional contribution of two hard gluons from production is obviously hopeless and not further discussed here. The fractions are converted into the cross section ratios as:

$$
\begin{aligned}
\sigma_{t t} / \sigma_{t t X} & =f_{t t} /(1-f b g), \\
\sigma_{t t j} / \sigma_{t t X} & =f_{t t j} /(1-f b g) .
\end{aligned}
$$

Limits at the $90 \%$ confidence level are also set on the cross section fractions:

$$
\begin{aligned}
& \sigma_{t t j} / \sigma_{t t X}\left(E_{T}=20 \mathrm{GeV}\right)<0.48, \\
& \sigma_{t t j} / \sigma_{t t X}\left(E_{T}=25 \mathrm{GeV}\right)<0.55 .
\end{aligned}
$$

Although the measurement lacks statistics, a method to determine the hard gluon radiation in top production has been established.

\section{RECENT RESULTS FROM BOTTOM PRODUCTION}

Bottom production is huge at the Tevatron. The total production cross section is on the order of about $100 \mu \mathrm{b}$ as compared to $1 \mathrm{nb}$ at the $\Upsilon(4 s)$ resonance or $7 \mathrm{nb}$ at the $Z$ resonance. Nevertheless $b$ physics is challenging at the Tevatron since the light quark inelastic cross section is another 3 orders of magnitude larger. 


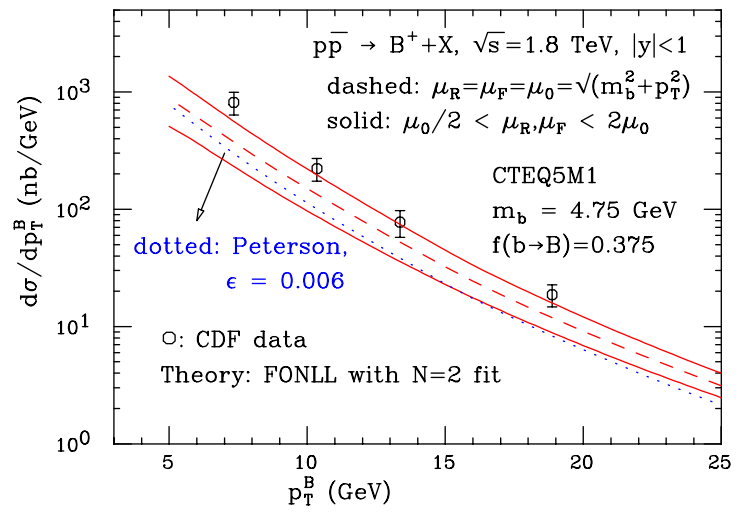

Figure 2. Differential production cross section of $b \bar{b}$ production at $1.8 \mathrm{TeV}$.

\subsection{Single Inclusive Cross Section}

A measurement of the single inclusive $b$ production is performed using the exclusive signal $B^{+} \rightarrow J / \psi K^{+}$with $J / \psi \rightarrow \mu^{+} \mu^{-}$[5]. Since the $b$ quark is not measured directly the $B$ meson measurement has to be unfolded for various effects like fragmentation, structure functions, momentum scales and $b$ quark mass. When cross section was compared to the predictions in the original publication it comes out too high by a factor of 2.9. Recent developments in the theory sector [6] shows that the Peterson fragmentation function [8] with an $\epsilon$ parameter value of $0.006 \pm 0.002$ is not appropriate to use in conjunction with next-to-leading order, NLO, programs [7]. This is due to the effect that the fragmentation parameter $\epsilon$ has been determined using leading-log calculations which are commonly used in shower Monte Carlo programs like Pythia [3].

The authors of Reference [6] suggest a more correct implementation of the fragmentation function to be used in connection with the NLO calculation. This new calculation is referred to as FONLL by the authors. Using the new scheme for the implementation of the fragmentation the cross section prediction is only a factor of 1.7 higher than the measured cross section. The situation is nicely summarized in Figure 2. The data points include statistical and systematic errors. The dashed line is the central value of the FONLL calculations while the solid lines indicate the theoretical uncertainties introduced by the variation of the momentum scales of renormalization and factorization. Also shown as a dotted line is the cross section prediction when using Peterson fragmentation with a parameter of $\epsilon=0.006$. The new calculation does not exhibit a large discrepancy to the measurements within the quoted uncertainties.

\subsection{Correlations of $b \bar{b}$ Production}

Recently CDF has started an effort to measure the correlations of $b \bar{b}$ production. Similar to the single inclusive cross section $b$ hadrons are used to identify the $b$ quark. Again an unfolding process has to be applied, only this time there are two $b$ hadrons, which makes the process much more complicated. From the measurement of $b \bar{b}$ correlations it is possible to distinguish contributions of different production mechanisms. The lowest order production mechanism is expected to produce $b \bar{b}$ pairs basically back-to-back while for the higher order production mechanisms the distribution of the angle between the two quarks is almost flat.

So far CDF has only results for correlations of $b$ hadrons which are not yet unfolded for detector effects. Two analyses have been performed.

In the first analysis an inclusive $J / \psi$ sample is used where the second $b$ hadron is identified by its semileptonic decay, a high $p_{T}$ lepton. The ratio of the numbers of events with the lepton in the same hemisphere with the $J / \psi$, over the number of events with the lepton in the opposite hemisphere is measured to be:

$$
R(\text { same/opposite })=0.52 \pm 0.20
$$

where the uncertainty is statistical and systematic. The measurement indicates that a large fraction of $b \bar{b}$ events have small angles between the two quarks and thus the data has contributions from higher order production mechanisms. The measurement suffers from low statistics, but $b$ hadrons are cleanly identified.

In the second analysis $b$ hadrons are identified by tagging secondary vertices in jets. This method has the advantage that there are many more events but at the cost of larger background. 
The $\Delta \phi$ angle between the two jets is shown in Figure 3 , and compared to the Pythia prediction at the lowest order. While data and Monte Carlo agree quite well for large opening angles it does not describe a significant fraction of events with small opening angle.

In summary, these first measurements indicate that there is a significant fraction of higher order production mechanisms present in the data, which can hopefully be disentangled in the near future.

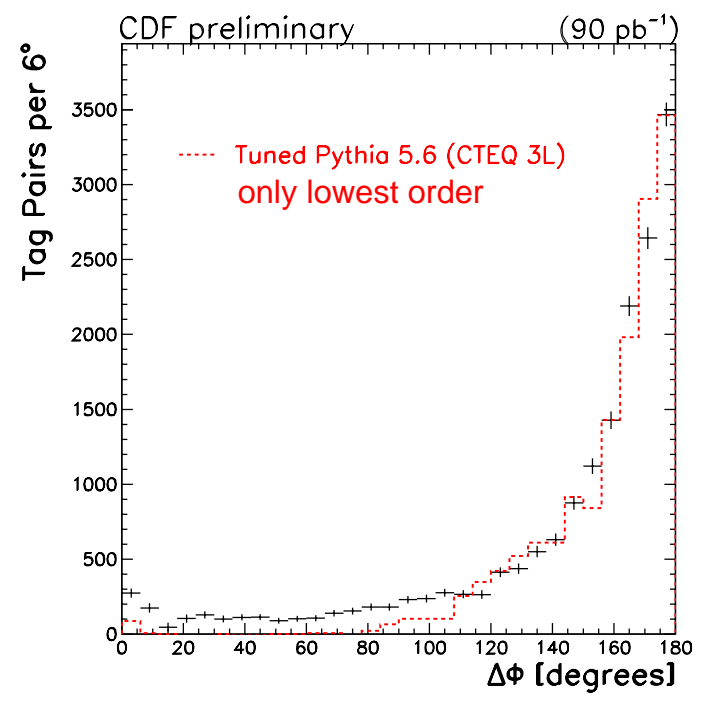

Figure 3. Smaller angle between the two $b$ jets, $\Delta \phi$ in the transverse plane. Dots with error bars are data.

\section{SEARCH FOR $\eta_{b}$}

An $\eta_{b}$ search is performed in about $80 \mathrm{pb}^{-1}$ of Run I data. The $\eta_{b}$ is searched for in its decay to two $J / \psi$ mesons, where the $J / \psi$ decays to a muon pair. The dataset is based on the dimuon $J / \psi$ trigger which constitutes the first $J / \psi$. Muons are identified by the trigger starting at about $2.2 \mathrm{GeV}$ with a noticeable turn-on curve. For the second $J / \psi$ the momentum cuts are significantly relaxed and the fourth muon need not be identified by the muon system.

The $\eta_{b}$ mass is expected to lie in a window from $9.36 \mathrm{GeV}$ to $9.46 \mathrm{GeV}$ according to various NRQCD predictions [9]. The analysis turns up 7 events in the given mass window with a background contribution of 1.8 events. The mass distribution is shown in Figure 4, where the expected mass window is indicated by the arrows. Observing 7 events with an expected background of 1.8 translates into a probability of $1.5 \%$ for this observation to be due to background; a 2.2 standard deviation effect. Assuming the events come from the signal the mass of the $\eta_{b}$ is determined to be:

$$
m\left(\eta_{b}\right)=9446 \pm 6 \mathrm{MeV} / c^{2} .
$$

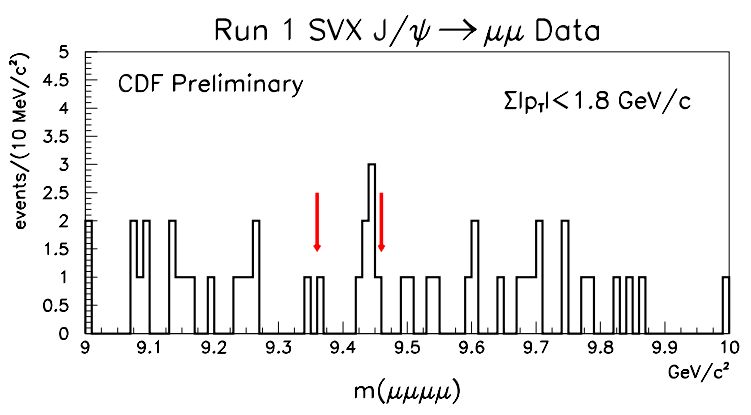

Figure 4. Mass distribution of the two $J / \psi$ mesons decaying to dimuons. The arrows indicate the region for the expected $\eta_{b}$ signal.

Run II data will allow us to significantly enlarge the data sample and if the signal is true a definite observation is soon possible.

\section{A GLIMPSE AT RUN II DATA}

Since April 2001 CDF has started its Run II data taking period with a significantly upgraded detector [10]. The most striking upgrade in terms of $b$ physics is certainly the trigger which allows us to trigger on high $p_{T}$ displaced tracks at the Level-2 trigger [1]. Also important are the upgrades of the silicon detector with higher 
vertexing precision and larger coverage, and the upgrade of the muon system with lower trigger thresholds and larger coverage.

\subsection{Detector Performance}

The performance of the CDF detector is already by now close to what has been achieved in the Run I data taking period. Since the $J / \psi$ trigger delivers a large signal and is well understood from Run I it is used to demonstrate CDF performance. The momentum scale calibration performed on this signal [11] allows us to measure $B$ meson masses. The masses of $B^{+}, B^{0}$ and $B_{s}^{0}$ mesons have been measured in their most prominent $J / \psi$ decay modes $B^{+} \rightarrow$ $J / \psi K^{+}, B^{0} \rightarrow J / \psi K^{* 0}\left(K^{* 0} \rightarrow K^{+} \pi^{-}\right)$and $B_{s}^{0} \rightarrow J / \psi \phi\left(K^{* 0} \rightarrow K^{+} K^{-}\right)$:

$$
\begin{aligned}
m\left(B^{+}\right) & =5280.6 \pm 1.7 \pm 1.1 \mathrm{MeV} / c^{2} \\
m\left(B^{0}\right) & =5279.8 \pm 1.9 \pm 1.4 \mathrm{MeV} / c^{2} \\
m\left(B_{s}^{0}\right) & =5360.3 \pm 3.8_{-2.9}^{+2.1} \mathrm{MeV} / c^{2}
\end{aligned}
$$

where the first uncertainty is the statistical and the second the systematic one. All measurements are in good agreement with the world averages and the measurements are statistically limited. The $B_{s}^{0}$ mass measurement is the second most precise single measurement and will soon be improved significantly. The $B_{s}^{0}$ mass distribution containing 14.4 signal events after background subtraction is shown in Figure 5.

Also the vertex resolution is quite well understood already. Therefore the inclusive $b$ hadron lifetime has been measured again using the inclusive $J / \psi$ sample. Furthermore, the $B^{+}$lifetime has been measured in its decay $B^{+} \rightarrow J / \psi K^{+}$. The results are:

$$
\begin{aligned}
& c \tau_{\text {incl }}=458 \pm 10 \pm 11 \mu \mathrm{m} \\
& c \tau_{B^{+}}=446 \pm 43 \pm 13 \mu \mathrm{m}
\end{aligned}
$$

where the first uncertainty is the statistical and the second the systematic one. Both measurements are in excellent agreement with the world average and the systematic uncertainties indicates the good understanding of the resolutions functions. In all analyses so far neither silicon hits with $z$ information nor the innermost silicon layer is used, because they are still being commissioned. A significant improvement in momentum

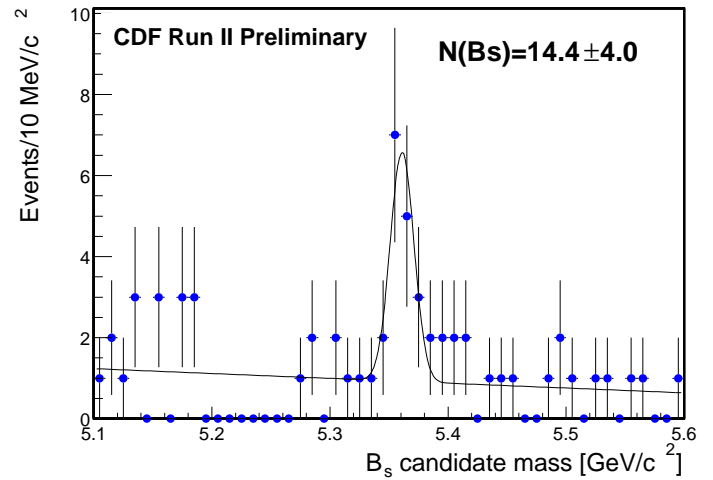

Figure 5. Mass distribution of the $B_{s}^{0}$ meson candidates in their decay $B_{s}^{0} \rightarrow J / \psi \phi\left(K^{* 0} \rightarrow\right.$ $\left.K^{+} K^{-}\right)$.

and vertexing resolution is expected once they are available.

\subsection{Charm Physics}

The commissioning of the displaced track trigger has enabled CDF to trigger on hadronic decay products of longer, lived particles like $b$ and $c$ hadrons. Although the lifetime of $b$ hadrons is longer the huge production cross section of charm quarks makes this trigger especially rich in $D$ mesons. This has opened a completely new window of opportunities for CDF: charm physics. In about $5 \mathrm{pb}^{-1}$ of data CDF sees about $30 \mathrm{k}$ $D^{0} \rightarrow K^{-} \pi^{+}, 6 \mathrm{k} D^{*+} \rightarrow D^{0}\left(K^{-} \pi^{+}\right) \pi^{+}, 27 \mathrm{k}$ $D^{+} \rightarrow K^{-} \pi^{+} \pi^{+}$and $1 \mathrm{k} D_{s}^{+} \rightarrow \phi\left(K^{+} K^{-}\right) \pi^{+}$. This is an impressive collection of $D$ mesons taking into account that the integrated luminosity will improve by more than 2 orders of magnitude.

We start a measurement of the charm production cross section. The first step is to identify background from secondary charm. This exercise is also necessary for doing $b$ physics with this trigger where the background and the signal are reversed.

To separate prompt from secondary charm the impact parameter of the fully reconstructed $D$ meson is used. Direct charm should point back to the primary vertex corresponding to a zero impact parameter. Secondary charm on the contrary originates from a $b$ hadron which has a long 
lifetime and has other decay products apart from the $D$ meson. Thus the $D$ does not necessarily point back to the primary vertex and its impact parameter distribution is rather wide. The detector resolution smears out both prompt and secondary charm impact parameter distributions.

The detector resolution function, $R$, is measured from the impact parameter distributions from $K_{s} \rightarrow \pi^{+} \pi^{-}$decays, which are predominantly prompt. This function should be the same for all decays. The inclusive impact parameter distributions of the $D$ mesons is than fitted to the function:

$$
\begin{aligned}
F\left(d_{0}\right)= & f_{B} \int F_{B}(x) R\left(d_{0}-x\right) d x+ \\
& +\left(1-f_{B}\right) R\left(d_{0}\right)
\end{aligned}
$$

where $f_{B}$ and $F_{B}$ are the fraction of secondary $D$ mesons and the impact parameter distribution of the secondary $D$ mesons. The variable $x$ integrates over all possible values of the impact parameter. For the fit the un-smeared impact parameter distribution for secondary $D$ mesons, $F_{B}$, is obtained from Monte Carlo. The impact parameter distribution is shown in Figure 6 with the fit superimposed indicating the contribution of the secondary $D$ mesons. As a comparison the resolution function for prompt $D$ mesons is assumed to be a simple Gaussian. The result of both fits for the fraction of $D$ mesons which originate from the decay of a $b$ hadron are quoted below, in parenthesis the numbers when using the simple Gaussian resolution function:

$$
\begin{array}{lll}
D^{0}: & 16.43 \pm 0.65 \% & (23.14 \pm 0.59 \%) \\
D^{*+}: & 11.41 \pm 1.37 \% & (20.00 \pm 1.19 \%) \\
D^{+}: & 11.26 \pm 0.53 \% & (17.29 \pm 0.53 \%) \\
D_{s}: & 34.80 \pm 2.75 \% & (37.84 \pm 2.60 \%)
\end{array}
$$

The difference bbetween both models give an impression of the uncertainty of this measurement. In general we conclude that the charm sample has contamination from secondary $D$ mesons between $10 \%$ and $40 \%$. The differences for the various $D$ mesons are due to the differing production fractions, lifetimes and the different number of decay products which have different probablities to pass the trigger.

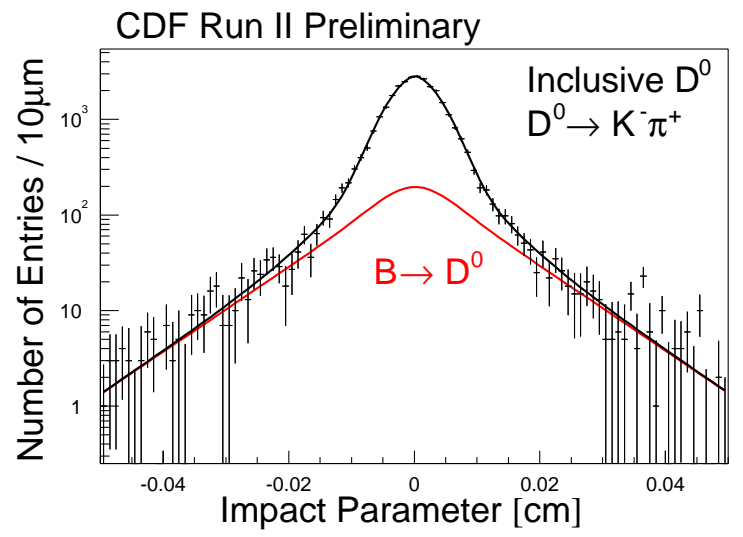

Figure 6. Impact parameter distribution for $D^{0}$ mesons in its decay $D^{0} \rightarrow K^{-} \pi^{+}$. The tails are mostly due to secondary $D^{0}$ decays while the center is mostly due to prompt $D^{0}$ decays. Overlayed is the fit described in the text.

\section{REFERENCES}

1. A. Cerri, in these proceedings.

2. The CDF collaboration, T. Affolder et al., Phys. Rev. D63 (2001) 032003.

3. H. U. Bengston and T. Sjöstrand, Comp. Phys. Comm. 43, (1987) 43; T. Sjöstrand, Comp. Phys. Comm. 82 (1994) 74.

4. D. Rainwater, private communiction.

5. The CDF Collaboration, D. Acosta et al., Phys. Rev. D65 (2002) 052005.

6. M. Cacciari and P. Nason, Phys. Rev. Lett $\mathbf{8 9}$ (2002) 122003.

7. P. Nason, S. Dawson and R.K. Ellis, Nucl. Phys. B303 (1988) 607; Nucl. Phys. B327 (1989) 49.

8. C. Peterson et al., Phys. Rev. D27 (1983) 105.

9. Most recent only: A. Spitz et al., hep/lat9906009, June 1999; T. Manke, Phys. Rev. D62 (2000) 114508; D.E. Groom et al., Eur. Phys. J. C15 (2000) 1.

10. The CDF II Collaboration, The CDF II Detector Technical Design Report, FERMILABPub-96/390-E (1996).

11. F. Bedeschi, in these proceedings. 